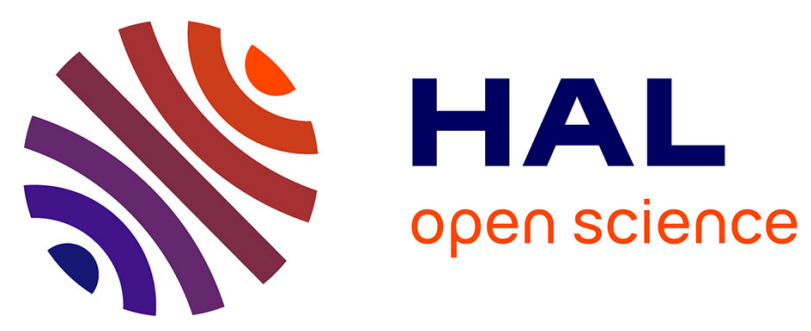

\title{
Invasive and in situ cervical cancer associated with pregnancy: analysis from the French cancer network (CALG: Cancer Associé à La Grossesse)
}

Anne Puchar, Anne-Sophie Boudy, Lise Selleret, A. Arfi, C. Owen, Sofiane Bendifallah, Emile Daraï

\section{To cite this version:}

Anne Puchar, Anne-Sophie Boudy, Lise Selleret, A. Arfi, C. Owen, et al.. Invasive and in situ cervical cancer associated with pregnancy: analysis from the French cancer network (CALG: Cancer Associé à La Grossesse). Clinical and Translational Oncology, 2020, 22 (11), pp.2002-2008. 10.1007/s12094020-02343-5 . hal-03009208

\section{HAL Id: hal-03009208 \\ https://hal.sorbonne-universite.fr/hal-03009208}

Submitted on 24 Nov 2020

HAL is a multi-disciplinary open access archive for the deposit and dissemination of scientific research documents, whether they are published or not. The documents may come from teaching and research institutions in France or abroad, or from public or private research centers.
L'archive ouverte pluridisciplinaire HAL, est destinée au dépôt et à la diffusion de documents scientifiques de niveau recherche, publiés ou non, émanant des établissements d'enseignement et de recherche français ou étrangers, des laboratoires publics ou privés. 
Invasive and in situ cervical cancer associated with pregnancy: Analysis from the French cancer network (CALG: Cancer Associé à La Grossesse).

Puchar A MD ${ }^{1}$, Boudy AS MD ${ }^{1}$, Selleret L MD ${ }^{1}$, Arfi A MD ${ }^{1}$, Owen C MD ${ }^{1}$, Bendifallah S MD ${ }^{1,2}$, PhD, Darai E MD, PhD ${ }^{1,2}$

1 Department of Gynaecology and Obstetrics, Tenon University Hospital, Assistance Publique des Hôpitaux de Paris (AP-HP), University Pierre-et-Marie-Curie Paris VI, Institut Universitaire de Cancérologie (IUC), France ; Centre CALG (Cancer Associé à La Grossesse), France.

2 UMRS-938 4. Faculté de Médecine Sorbonne Université, France.

Study conducted in Tenon University Hospital, Paris, France

Corresponding author: Anne Puchar. Department of Gynaecology and Obstetrics, Tenon University Hospital, Assistance Publique des Hôpitaux de Paris (AP-HP), University Pierreet-Marie-Curie Paris VI, Institut Universitaire de Cancérologie (IUC), France ; Centre CALG (Cancer Associé à La Grossesse), France. +33156016019

anne.puchar@gmail.com 


\section{$\underline{\text { Abstract }}$}

Purpose: To describe the oncologic and obstetric outcomes of patients diagnosed with invasive cervical cancer (ICC) and in situ adenocarcinoma (ISA) during pregnancy or during the year following delivery.

Methods: This retrospective observational study involved a cohort of 28 patients diagnosed with invasive cervical cancer (20 patients) or in situ adenocarcinoma (8 patients) during pregnancy or during the year following delivery who received expert opinion from physicians of the CALG (Cancer Associé à La Grossesse) network between 2005 and 2018. Descriptive results were expressed in median, range and interquartile range (IQR).

Results: Between 2005 and 2018, 20 patients with ICC and eight with ISA received expert opinion from physicians of the CALG network. Both ICC and ISA were mostly diagnosed during pregnancy with a median term at diagnosis of 23.3 weeks of gestation (WG) for ICC and 7.3 WG for ISA. Overall, the median age at diagnosis for both ICC and ISAwas33 years. Most ICCs ( $\mathrm{n}=9$ ) had FIGO stage $\geq$ IB2 and five underwent neoadjuvant chemotherapy at a median term of 22.5 WG. Seventeen patients with ICC underwent surgery. Three patients had medical termination of the pregnancy. Two patients experienced recurrence and three died. Median time of follow-up was 59.3 months (IQR 30.5-129.2).

Conclusion: Management of cervical cancer during pregnancy is challenging especially in terms of maternal outcomes with a relative poor prognosis requiring a multidisciplinary expert advice. 
Keywords: Cervical cancer; Pregnancy; Pregnancy-associated cervical cancer; Prognosis; chemotherapy. 


\section{INTRODUCTION}

Cervical cancer is defined as being associated with pregnancy when diagnosed during pregnancy or during the year following delivery.[1] Cervical cancer remains the fourth most common cancer in women of childbearing age after breast cancer, melanoma and thyroid cancer[2], even though prevention by Human Papillomavirus (HPV) vaccination and screening for cervical cancer has greatly improved in recent years[3].

Although cervical cancer during pregnancy is rare, with an estimated incidence of 12 to 15 per 100.000 pregnancies[4,5], an increase in the number of cases has been observed during the last two decades particularly because of delayed childbearing[6]. It constitutes a major medical challenge related to the impact of treatment on both maternal and fetal outcomes. In the absence of randomized trials guiding treatment, the management of cervical cancer during pregnancy is based on expert consensus taking into account gestational age, stage at diagnosis, the desire to continue the pregnancy, and the trade-off between optimal effective treatment and preservation of the health of the fetus. These women should thus be followed by an expert center.

The aim of the current study was to describe the oncologic and obstetric outcomes of patients diagnosed with invasive cervical cancer (ICC) and in situ adenocarcinoma (ISA) during pregnancy or during the year following delivery. 


\section{PATIENTS AND METHODS}

The CALG network was created in France by funding from the Institut National du Cancer (INCa) mainly to optimize management of patients with cancer associated pregnancy. Data of women with histologically proven ICC and ISA recorded in the prospective database of the CALG network between June 2005 and July 2018 were retrospectively analyzed. We included all patients who were treated in Tenon University Hospital or whose treating physician had consulted the CALG network.

The extent of the pelvic tumor was assessed by clinical examination and magnetic resonance imaging (MRI). The 2018 FIGO classification based on MRI was used for ICC.[79]

The following data were recorded: epidemiologic data; circumstances of diagnosis; histologic data; and treatment details. The type of surgery, term and mode of delivery were also recorded as were modalities of NACT and adjuvant chemotherapy and adjuvant therapy.

The Ethical Review Committee (CEROG) approved this study (CEROG 2019-GYN603). Written informed consent was obtained from all patients.

Statistical analysis was performed with R Studio version 3.5.2. Descriptive results were expressed in median, range and interquartile range (IQR). 


\section{RESULTS}

\subsection{Epidemiologic characteristics of the population}

Between June 2005 and July 2018, 20 patients with ICC and eight with ISA were recorded in the database of the CALG network. Characteristics of the patients are summarized in Table 1.

\subsection{Patients with ICC}

Of the 20 patients, twelve were treated in Tenon University Hospital. Eighteen cases were diagnosed during pregnancy at a median term of 23.3 weeks of gestation (WG) [min7max34.3; IQR $(17.1 ; 28.0)]$ and two during the year following delivery (8 weeks post-partum in both cases). The median age at diagnosis was 33 years old [min26-max42; IQR (29.0; 36.8)]. Five patients were current smokers, none were HIV positive.

Eleven of the 20 ICCs were squamous-cell carcinomas, eight were invasive adenocarcinomas and one was an undifferentiated carcinoma. Histologic type and FIGO classification of the ICCs are shown in Table 2.Most of the patients $(n=14)$ were stage $\geq$ IB2 at diagnosis. Four $(22.2 \%)$ patients underwent a PET-scan, one during pregnancy at 28 WG. Sixteen patients (80\%) underwent MRI, 13 during pregnancy.

\subsubsection{Medical and surgical management of patients with ICC}

Five patients underwent NACT (Table 3) at a median term 22.0 WG [min18.7$\max 23.6$; IQR $(20.4 ; 22.8)]$. 
Patient 1: The exact FIGO stage was not evaluable for this patient as the MRI at $22 \mathrm{WG}$ was normal and no clinical exam details were available. This patient was diagnosed with invasive adenocarcinoma at 19 WG following a cervical smear test HSIL HPV 18+. She received four cycles of carboplatin and taxol. She had a cesarean delivery (C-section) at $37+1 \mathrm{WG}$ and underwent radical hysterectomy. Histologic findings did not show any tumor residue.

Patient 2: This patient was diagnosed with squamous-cell carcinoma at 13 WG with a FIGO IB1 stage on MRI. She underwent a conization and cervical cerclage at $13+6$ WG. Histologic findings showed a moderately differentiated squamous-cell carcinoma of 16x10x5 mm with embol. She began chemotherapy with carboplatin and taxol at 22 WG. She underwent a Csection at $32 \mathrm{WG}$ with concomitant radical hysterectomy and pelvic lymphadenectomy. No tumor residue was found. None of the 17 lymph nodes removed were positive.

Patient 3: In this patient a FIGO IB2 stage invasive adenocarcinoma was diagnosed at $20 \mathrm{WG}$. MRI revealed a lesion of $35 \mathrm{~mm}$. She underwent NACT with carboplatin and paclitaxel at 23+4 WG. MRI reassessment found a tumor residue of $17 \mathrm{~mm}$. She had a C-section at $37 \mathrm{WG}$ and underwent radical hysterectomy with pelvic lymphadenectomy by laparoscopy 2 weeks later. Histologic findings showed a 25x26x9 mm tumor residue of invasive adenocarcinoma p16 + p53 30\%.Of the 24 lymph nodes removed, none were positive.

Patient 4: The fourth patient was diagnosed with IB3 stage squamous-cell carcinoma at 21 WG. The MRI found a lesion of $65 \mathrm{~mm}$. The patient refused medical pregnancy termination. She had two cycles of NACT by taxol and carboplatin. Reassessment showed local progression and revealed emergence of a dilatation of the right kidney cavities and bilateral external iliac lymph nodes. She underwent a C-section at $28 \mathrm{WG}$ with pelvic and para-aortic lymphadenectomy. Out of the 15 para-aortic lymph nodes removed, five were positive. She received concomitant radio-chemotherapy. The patient continued to progress despite treatment and died 4 months after diagnosis. 
Patient 5: The last patient underwent NACT for aIB2 stage squamous-cell carcinoma diagnosed at $25+6 \mathrm{WG}$. Initial MRI found a lesion of $32 \times 24 \times 27 \mathrm{~mm}$. She began chemotherapy with carboplatin and taxol. MRI reassessment found a tumor residue of 18x6x14 mm and positron emission tomography (PET) scan showed a partial metabolic response. She underwent radical hysterectomy, pelvic and para-aortic lymphadenectomy concomitant to a C-section. No data on final histology was available.

Eighteen patients (80\%) with ICC underwent surgery (Table 3).One patient with IB1 stage adenocarcinoma underwent pelvic lymphadenectomy by laparoscopy during the second trimester of pregnancy. No details of the lymph node status or surgical and pregnancy outcomes were available.

One patient with a IIB stage squamous-cell carcinoma underwent a retroperitoneal para-aortic lymphadenectomy at $11+5 \mathrm{WG}$. None of the 18 lymph nodes removed were positive. She refused medical pregnancy termination and received concomitant radio-chemotherapy resulting in uteri fetal death at $17+4$ WG. She underwent a completion radical hysterectomy 6 weeks after radiotherapy. Histology did not find tumor residue.

Three patients underwent medical termination of pregnancy: one at $30 \mathrm{WG}$ because of myelomeningocele diagnosed in utero and the two others during the first trimester for FIGO IB2 and IIIC1.Of the 18 patients with ICC diagnosed during pregnancy, eight gave birth to healthy children by C-section. Median term at delivery was 37 WG (IQR 35.5; 37). Of the remaining 10 patients, three had a medical termination of pregnancy, and one had a fetal death in utero. No data on pregnancy outcomes were available for the six remaining patients treated out of Tenon Hospital.

\subsubsection{Follow-up and recurrence in patients with ICC}


The median time of follow-up was 51.2months (IQR 24.5; 156.4). Two patients experienced recurrence and three died of the disease. In the first case, recurrence occurred 4 years after diagnosis of an adenocarcinoma on the Troisier lymph node; she died 6.5 years after the first diagnosis. In the second case, the patient was diagnosed at $33+2$ WG with welldifferentiated IB1 stage squamous-cell carcinoma. She underwent radical hysterectomy and pelvic lymphadenectomy 4 weeks after C-section at 37 WG. Out of the 10 pelvic lymph nodes removed, one was positive. The patient underwent para-aortic lymphadenectomy one month later removing 18 negative lymph nodes. Final FIGO stage was IIIC1. She received adjuvant radiotherapy and chemotherapy with a carboplatin-taxol regimen followed by paclitaxel with cisplatin. She relapsed at the iliac level 3 months after radical hysterectomy and pelvic lymphadenectomy and died 10 months after initial diagnosis.

The third patient underwent NACT for a differentiated IB3 squamous cell carcinoma at 21 WG. She had two cycles of NACT by taxol and carboplatin. Reassessment showed local progression and the patient underwent a C-section at $28 \mathrm{WG}$ with pelvic and para-aortic lymphadenectomy. The patient continued to progress despite treatment and died 4 months after diagnosis.

\subsection{Patients with ISA}

Of the eight patients, four were treated in Tenon Hospital. Five were diagnosed on biopsy and the three others on conization because of suspicion of cervical intraepithelial neoplasia (CIN) 2 or 3 on biopsy. Four were diagnosed during pregnancy with a median term at diagnosis of 7.3 WG [min 6.1-max 8; IQR $(6.7 ; 7.7)]$; four were diagnosed in the year following delivery. None were HIV positive. Median age at diagnosis was 33 years old [min 26-max 43; IQR $(30.3 ; 34.5)]$. One patient was a current smoker. All the patients underwent surgery. Two of these had a hysterectomy: one after conization followed by trachelectomy, 
and the second in a 43-year-old patient who refused fertility sparing surgery. The six remaining patients underwent a conization including one during pregnancy at a term of $10+6$ WG. Table 4 summarizes the surgical management of the women with ISA. Median term of follow-up was 79.00 months (IQR 63.1; 103.7) without recurrence. 


\section{DISCUSSION}

The present study reports the experience of the CALG network on the management of cervical cancer associated with pregnancy and confirms the possibility of maintaining the pregnancy in spite of a relatively high rate of recurrence and death.

In our population, 55\% had squamous-cell carcinoma and $40 \%$ had adenocarcinoma. This distribution of histologic types is in agreement with those of the meta-analysis of Halaska et al[10] but differs from those reported in a literature review by Song et al[11]who reported that $73.8 \%$ of the women (45 of 61 ) were diagnosed with squalors-cell carcinoma and $14.8 \%$ with invasive adenocarcinoma. Similarly, Kärrberg et al[12]reported rates of 76.6 $\%$ (36 of 47) and 17\% (8) for squamous-cell carcinoma and adenocarcinoma, respectively. This apparent discrepancy cannot be related to differences in the epidemiologic characteristics: our population was similar to those of previous studies with most patients being Caucasian $[10,11]$ but by difficulties in detecting adenocarcinoma. Several publications suggest that screening is less effective for adenocarcinoma than for squamous-cell carcinoma of the cervix[13-16]. This is because glandular lesions are mainly located in the cervical canal making sampling difficult and thus reducing the chance of early detection[15,17,18]. Moreover, several authors reported a lower accuracy of colposcopy in pregnant than in nonpregnant women[19,20]. Finally, Ciavattini et al observed a better reliability of colposcopy in the two first trimesters and especially before $20 \mathrm{WG}$ while the median term at diagnosis was 22.3 WG in the current study.

In contrast to ISA, which was often diagnosed during the first trimester of pregnancy, most of the patients with ICC had a FIGO stage $\geq$ IB2 and were mainly diagnosed during the second half of the second trimester of pregnancy. Halaska et al[10] recently published a multicenter matched cohort that gathered data from six European centers between 1990 and 2012 with a total of 132 pregnant patients. Their data were similar to our study with 
a median age at diagnosis of 34 years but a median gestational age at diagnosis slightly earlier at $18.4 \mathrm{WG}$, and with most patients $(80.3 \%)$ being diagnosed with early stage disease. However, FIGO staging was essentially based on clinical examination, with a risk of misdiagnosis as previously demonstrated[21,22], while in the current study MRI was often performed [11]. In 2018, the FIGO classification was revised to take into account imaging and pathologic findings[9]. The contribution of imaging in this new classification is a major asset in the staging of cervical cancer associated with pregnancy. Physiologic changes, especially the presence of cervical edema related to pregnancy, might alter the clinical assessment of tumor size[23] and MRI can be safely performed during pregnancy not only to evaluate the initial FIGO stage but also to determine response to chemotherapy and hence to adapt therapy[21,22]. Moreover, patients with lesions under $2 \mathrm{~cm}$ are eligible for conization or simple trachelectomy (used in $21 \%$ of our population) which appears to be a safe and effective treatment during pregnancy [24-26].

In the current study, excluding the patient with a myelomeningocele diagnosed in utero, only two patients $(11 \%)$ underwent a medical termination for advanced stage disease (IIB). This is a lower rate than that noted in Halaska et al study(26.5\%)[10] and suggests that continued pregnancy is possible for most women with cervical cancer associated with pregnancy. Moreover, Perrone et al[27] reported that termination of pregnancy was refused by two-thirds of patients even in the case of advanced cervical cancer. This raises the issue of NACT during pregnancy. After exclusion of the three patients undergoing pregnancy termination and the two patients diagnosed in the postpartum period, NACT was used in onethird of our population. Song et al[11]published a meta-analysis including 39 studies published between 1997 and 2018 including 88 patients with cervical cancer associated with pregnancy treated by platinum-based chemotherapy. In their meta-analysis, $87.5 \%$ of the patients were diagnosed with early stage (I and IIA - FIGO 2009) and $12.5 \%$ with advanced 
stage (IIB, III and IV) disease. Eighty-eight babies were delivered including 71 completely healthy neonates. In 2018, the ESGO-ESTRO-ESP [28]guidelines point out that, in view of the low incidence of cervical cancer during pregnancy and the lack of high level of evidence, patients should be exclusively managed in expert centers with the advice of a multidisciplinary committee taking into account gestational age, FIGO stage, the maternal and fetal risk-benefit ratio of each option, and the desire of the patient to maintain the pregnancy. No cancer treatment or surgical staging is proposed before 12 WG[29-31]. Over this term, surgery is recommended for early stage disease (Stage Ia-Ib1) and NACT for advanced stages (Ib2-IIa) [27].

Nevertheless, it should not be forgotten that cervical cancer associated with pregnancy is a severe disease. In the present study, two recurrences and three deaths were observed. Song et al[11]reported recurrence in $19.8 \%$ of patients, $90 \%$ of whom died from the disease. Similarly, Halaska et al[10] reported a recurrence rate of $20 \%$ and a death rate of $14 \%$ among pregnant women similar to that observed for non-pregnant women (21\% of recurrences and $18 \%$ of deaths).

In our study, the rate of surgery during pregnancy, NACT, expectant management till delivery, and premature delivery before treatment were 59\%, 29\%, $12 \%$ and $12 \%$, respectively. Our data are partially in contrast with those of Halaska et al[10]reporting a $17.4 \%$ rate of surgery, $16.7 \%$ of NACT, $26.5 \%$ of expectant management, and $12.9 \%$ of premature delivery[10]. This apparent discrepancy can be explained by the long study period of Halaskaet al's cohort and variations according to countries.

Some limits of the current study deserve to be underlined. First, the retrospective nature cannot exclude all biases. Second, the low sample size linked to the low incidence of 
the disease could be a source of misinterpretation. However, it is clear that the lower incidence of pregnancy termination and higher number of NACT administered compared to previous studies confirms the trend for a more conservative management of pregnancy which is also probably linked to delayed pregnancy in western countries. Finally, although all the women included were followed either by the CALG network or by physicians who sought advice from the network, some physicians contributed to analysis bias by failing to report some data especially about treatment options and follow-up.

\section{CONCLUSION}

This study reports the experience of the CALG network on the management of cervical cancer associated with pregnancy and supports a more conservative management of cervical cancer associated with pregnancy. 


\section{Ethics approval and consent to participate:}

The Ethical Review Committee (CEROG) approved this study (CEROG 2019-GYN-603). Written informed consent was obtained from all patients.

The study was performed in accordance with the Declaration of Helsinki.

\section{Consent for publication:}

Our manuscript doesn't contain any individual person's data in any form (including individual details, images or videos).

\section{Data availability:}

Data supporting the results reported in the article can be found by requested at the corresponding author.

\section{Conflict of interest:}

The authors declare no conflict of interest.

\section{Funding:}

This research did not receive any specific grant from funding agencies in the public, commercial, or not-for-profit sectors. 


\section{Authorship:}

Puchar A made the analysis and wrote the article.

Boudy AS implement the database and helped write the manuscript.

Selleret L, Arfi A and Owen C implement the database.

Bendifallah $\mathrm{S}$ helped write the manuscript.

Darai E helped write the manuscript. 


\section{REFERENCES}

[1] Petrek JA. Breast cancer during pregnancy. Cancer. 1994;74:518-527.

[2] McCormick A, Peterson E. Cancer in Pregnancy. Obstet. Gynecol. Clin. North Am. 2018;45:187-200.

[3] Mitchell K, Saraiya M, Bhatt A. Increasing HPV Vaccination Rates Through National Provider Partnerships. J. Womens Health 2002. 2019;

[4] Smith LH, Danielsen B, Allen ME, et al. Cancer associated with obstetric delivery: results of linkage with the California cancer registry. Am. J. Obstet. Gynecol. 2003;189:1128-1135.

[5] Eibye S, Kjær SK, Mellemkjær L. Incidence of Pregnancy-Associated Cancer in Denmark, 1977-2006: Obstet. Gynecol. 2013;122:608-617.

[6] Mathews TJ. First Births to Older Women Continue to Rise. 2014;8.

[7] Thomeer MG, Gerestein C, Spronk S, et al. Clinical examination versus magnetic resonance imaging in the pretreatment staging of cervical carcinoma: systematic review and meta-analysis. Eur. Radiol. 2013;23:2005-2018.

[8] Mitchell DG, Snyder B, Coakley F, et al. Early invasive cervical cancer: tumor delineation by magnetic resonance imaging, computed tomography, and clinical examination, verified by pathologic results, in the ACRIN 6651/GOG 183 Intergroup Study. J. Clin. Oncol. Off. J. Am. Soc. Clin. Oncol. 2006;24:5687-5694.

[9] Bhatla N, Aoki D, Sharma DN, et al. Cancer of the cervix uteri. Int. J. Gynecol. Obstet. 2018;143:22-36.

[10] Halaska MJ, Uzan C, Han SN, et al. Characteristics of patients with cervical cancer during pregnancy: a multicenter matched cohort study. An initiative from the International Network on Cancer, Infertility and Pregnancy. Int. J. Gynecol. Cancer Off. J. Int. Gynecol. Cancer Soc. 2019;

[11] Song Y, Liu Y, Lin M, et al. Efficacy of neoadjuvant platinum-based chemotherapy during the second and third trimester of pregnancy in women with cervical cancer: an updated systematic review and meta-analysis. Drug Des. Devel. Ther. 2019;13:79-102.

[12] Kärrberg C, Rådberg T, Holmberg E, et al. Support for down-staging of pregnancyassociated cervical cancer. Acta Obstet. Gynecol. Scand. 2015;94:654-659.

[13] Pettersson BF, Hellman K, Vaziri R, et al. Cervical cancer in the screening era: who fell victim in spite of successful screening programs? J. Gynecol. Oncol. 2011;22:7682.

[14] Zappa M, Visioli CB, Ciatto S, et al. Lower protection of cytological screening for adenocarcinomas and shorter protection for younger women: the results of a casecontrol study in Florence. Br. J. Cancer. 2004;90:1784-1786. 
[15] Castanon A, Landy R, Sasieni PD. Is cervical screening preventing adenocarcinoma and adenosquamous carcinoma of the cervix? Int. J. Cancer. 2016;139:1040-1045.

[16] Mancini S, Ravaioli A, Giuliani O, et al. Incidence and survival trends of cervical adenocarcinoma in Italy: Cytology screening has become more effective in downstaging the disease but not in detecting its precursors. Int. J. Cancer. 2017; 140:247-248.

[17] Davy MLJ, Dodd TJ, Luke CG, et al. Cervical cancer: effect of glandular cell type on prognosis, treatment, and survival. Obstet. Gynecol. 2003;101:38-45.

[18] Pirog EC. Cervical Adenocarcinoma: Diagnosis of Human Papillomavirus-Positive and Human Papillomavirus-Negative Tumors. Arch. Pathol. Lab. Med. 2017;141:16531667.

[19] Morimura Y, Fujimori K, Soeda S. Cervical cytology during pregnancy _ comparison with non-pregnant women and management of pregnant women with abnormal cytology. Fukushima J Med Sci 2002 Jun48127e37 PubMed PMID 12365596.

[20] La Russa M, Jeyarajah AR. Invasive cervical cancer in pregnancy. Best Pract. Res. Clin. Obstet. Gynaecol. 2016;33:44-57.

[21] Clinical examination versus magnetic resonance imaging in the pretreatment staging of cervical carcinoma: systematic review and meta-analysis. Eur Radiol 2013 2320052018.

[22] Donald G. Mitchell, Bradley Snyder, Fergus Coakley, Caroline Reinhold, Gillian Thomas, Marco Amendola,, Lawrence H. Schwartz, Paula Woodward, Harpreet Pannu, and Hedvig Hricak. Early Invasive Cervical Cancer: Tumor Delineation by Magnetic Resonance Imaging, Computed Tomography, and Clinical Examination, Verified by Pathologic Results, in the ACRIN 6651/GOG 183 Intergroup Study. J Clin Oncol 245687-5694 (C) 2006 Am. Soc. Clin. Oncol.

[23] Michael CW, Esfahani FM. Pregnancy-related changes: a retrospective review of 278 cervical smears. Diagn. Cytopathol. 1997;17:99-107.

[24] Salvo G, Frumovitz M, Pareja R, et al. Simple trachelectomy with pelvic lymphadenectomy as a viable treatment option in pregnant patients with stage IB1 $(\geq 2 \mathrm{~cm})$ cervical cancer: Bridging the gap to fetal viability. Gynecol. Oncol. 2018;150:50-55.

[25] Umemoto $\mathrm{M}$, Ishioka S, Mizugaki $\mathrm{Y}$, et al. Obstetrical prognosis of patients who underwent vaginal radical trachelectomy during pregnancy. J. Obstet. Gynaecol. Res. 2019;

[26] Yoshihara K, Ishiguro T, Chihara M, et al. The Safety and Effectiveness of Abdominal Radical Trachelectomy for Early-Stage Cervical Cancer During Pregnancy. Int. J. Gynecol. Cancer Off. J. Int. Gynecol. Cancer Soc. 2018;28:782-787.

[27] Perrone AM, Bovicelli A, D'Andrilli G, et al. Cervical cancer in pregnancy: Analysis of the literature and innovative approaches. J. Cell. Physiol. 2019; 
[28] Cibula D, Pötter R, Planchamp F, et al. The European Society of Gynaecological Oncology/European Society for Radiotherapy and Oncology/European Society of Pathology Guidelines for the Management of Patients With Cervical Cancer. Int. J. Gynecol. Cancer Off. J. Int. Gynecol. Cancer Soc. 2018;28:641-655.

[29] Cardonick E, Iacobucci A. Use of chemotherapy during human pregnancy. Lancet Oncol. 2004;5:283-291.

[30] Köhler C, Oppelt P, Favero G, et al. How much platinum passes the placental barrier? Analysis of platinum applications in 21 patients with cervical cancer during pregnancy. Am. J. Obstet. Gynecol. 2015;213:206.e1-5.

[31] Pereg D, Koren G, Lishner M. Cancer in pregnancy: gaps, challenges and solutions. Cancer Treat. Rev. 2008;34:302-312. 